\title{
Incremento na mortalidade associada à presença de diabetes mellitus em nipo-brasileiros
}

\section{Increase in the mortality associated with the presence of diabetes mellitus Japanese-Brazilians}

Suely G odoy A. G imeno, Sandra R.G. Ferreira, Laércio Joel Franco, Magid Iunes, Katsumi 0 siro e Grupo de Estudo de Diabetes em Nipo-Brasileiros*

Departamento de Medicina Preventiva da U niversidade Federal de São Paulo (U NIFESP-EPM). São Paulo, SP

GIM EN O Suely Godoy A. Incremento na mortalidade associada à presença de diabetes mellitus em nipo-brasileiros Rev. Saúde Pública, 32 (2): 118-24, 1998

(c) Copyright Faculdade de Saúde Pública da U SP. Proibida a reprodução mesmo que parcial sem a devida autorização do Editor Científico. Proibida a utilização de matérias para fins comerciais. All rights reserved. 


\title{
Incremento na mortalidade associada à presença de diabetes mellitus em nipo-brasileiros
}

\author{
Increase in the mortality associated with the presence of \\ diabetes mellitus Japanese-Brazilians
}

\author{
Suely G odoy A. G imeno, Sandra R.G. Ferreira, Laércio Joel Franco, Magid Iunes, Katsumi \\ $\mathbf{O}$ siro e $\mathbf{G}$ rupo de Estudo de Diabetes em N ipo-Brasileiros*
}

Departamento de Medicina Preventiva da Universidade Federal de São Paulo (U NIFESP-EPM). São Paulo, SP - Brasil

Objetivo

Material e Método

Resultados e Conclusões

\begin{abstract}
Resumo
Como parte de um estudo envolvendo migrantes japoneses (issei) e seus descendentes (nisei), residentes na cidade de Bauru no Estado de São Paulo, descrevem-se e comparam-se os coeficientes de mortalidade (CM) observados para o período de 1993 a 1996 em indivíduos com graus diferentes de tolerância à glicose.
\end{abstract}

Nesse estudo, em 1993, a coorte era composta por 530 nipo-brasileiros (236 issei e 294 nisei), de ambos os sexos, com idade entre 40 e 79 anos, sendo que 91 indivíduos (17\%) foram classificados como diabéticos não dependentes de insulina (DMNDI), 90 (17\%) como portadores de tolerância à glicose diminuída (TGD) e 349 (66\%) como normais quanto à tolerância à glicose. Em 1996 foram identificados os óbtos ocorridos e obtidas informações dos familiares e dos certificados de óbito para o registro da data e da causa da morte. Calcularam-se, para os três grupos de indivíduos, os CM brutos e ajustados, por todas as causas e por causas específicas (doenças circulatória e renal). O modelo de Cox foi utilizado para a comparação dos CM ajustados segundo idade, sexo, geração, creatinina sérica, presença de hipertensão arterial, de dislipidemia e de obesidade.

As razões entre os CM brutos de indivíduos diabéticos e normais foram 2,95 (IC 95\%: 1,10 -7,62) para os óbitos ocorridos por todas as causas e 4,75 (IC 95\%: 1,31 - 16,48) para os óbitos por causas específicas. Não foram observadas diferenças estatisticamente significantes entre os CM brutos de indivíduos com TGD quando comparados aos indivíduos normais. Após o ajuste simultâneo pelas variáveis de controle, observou-se que, entre os indivíduos diabéticos, a força de mortalidade por causas específicas foi aproximadamente 4 vezes aquela observada entre os indivíduos normais (Razão dos CM: 3,86 e IC 95\%: 1,11 -13,38). Os resultados em nipo-brasileiros são consistentes com outros

\footnotetext{
*As seguintes pessoas participaram do Grupo de Estudo de Diabetes em Nipo-Brasileiros: Amélia T. Hirai, Lúcia C. Iochida, Luiza K. Matsumura, Regina Santiago Moisés, Sérgio A. Dib, Alcides Hirai e Marco Antonio Vivolo (UNIFESP); Mário Kikushi, Nobue Miyasaki, Ênio Wakisaka (USP); Katsunori Wakisaka (Centro de Estudos Nipo-Brasileiros); Cana Taniguchi (Secretaria da Saúde ESP); Helena C. F. Oliveira (UNICAMP); Nilce Tomita (USP/Bauru); Rita Chaim (Universidade Sagrado Coração-Bauru); José Alberto S. Freitas (HRLLPB-USP) e Akira Ishikiriama (Clube-Nipo Brasileiro de Bauru). Correspondência para / Correspondence to: Suely Godoy Agostinho Gimeno - Rua Botucatu, 740 Vila Clementino - 04023-062 -Brasil - São Paulo, SP. Email: suely@medprev.epm.br

Edição subvencionada pela FAPESP (Processo n 97/09815-2).

Recebido em 7.4.1997. Aprovado em 20.8.1997.
} 
obtidos em populações diabéticas, reforçando a influência desse distúrbio metabólico, particularmente sobre a mortalidade por doenças cardiovascular e renal.

Coeficiente de mortalidade. Diabetes mellitus.

\begin{abstract}
Objective

Research design and Method

Results and Conclusions
\end{abstract}

\section{INTRO DU ÇÃO}

Em todo o mundo, o impacto do diabetes mellitus (DM) está refletido em morbidade aumentada e em mortalidade prematura ${ }^{21}$. Hanis e col. ${ }^{8}$ afirmam que, nos Estados Unidos, a esperança de vida de homens e mulheres com DM é reduzida em aproximadamente nove e sete anos, respectivamente. Esses autores destacaram que, apesar de numerosos estudos terem mostrado a existência de associação de DM com a morbidade e com a mortalidade, as investigações sobre a mortalidade têm sido prejudicadas devido às limitações inerentes aos registros vitais. Laurenti e col. ${ }^{11}$, em estudo sobre a mortalidade por DM no Município de São Paulo, destacaram que os dados oficiais apresentaram razoável fidedignidade para o estudo do DM como causa básica de morte, mas não se prestaram à análise como causa múltipla, uma vez que a importância do DM foi fortemente subestimada.

Franco e col. ${ }^{4}$, em estudo sobre causas múltiplas de óbitos, observaram que do total de 202.141 óbitos ocorridos no ano de 1992 no Estado de São Paulo, o DM foi mencionado em 13.786 certificados $(6,8 \%)$, sendo causa básica em 5.303 (2,6\%). Nos casos onde o DM figurou como causa associada, as principais causas básicas foram: doenças cardiovasculares 
$(62,9 \%)$, respiratórias $(12,6 \%)$ e neoplásicas $(6,9 \%)$. Situação semelhante a essa foi descrita por Melo e col. ${ }^{15}$ para a cidade de Recife. Apesar da subestimação da mortalidade por DM, os registros vitais têm sistematicamente indicado que o DM é problema de saúde pública importante em diferentes populações em todo o mundo ${ }^{6,13,17}$.

Da mesma forma que para população adulta em geral, a mortalidade em pessoas com DM não dependente de insulina (DMNDI) aumenta com a idade. Contudo, entre indivíduos diabéticos, a força de mortalidade é o dobro daquela observada entre aqueles que não têm essa doença ${ }^{6}$. Acredita-se que a elevação da mortalidade nos indivíduos com DM ocorra não apenas em decorrência de suas complicações macro e microvasculares, mas também pela sua associação com outras condições mórbidas tais como a obesidade, a hipertensão arterial e a dislipidemia ${ }^{1,10}$.

Como em outras doenças, onde a exposição a fatores exógenos tem relevância etiopatogênica, o perfil de morbimortalidade por DM de uma população se altera segundo o lugar: quando indivíduos se deslocam de uma região de baixo risco para uma de maior risco, eles assumem a condição do novo ambiente. Gotlieb ${ }^{7}$, em 1990, em estudo feito com issei residentes no Município de São Paulo, encontrou, para o ano de 1980, um coeficiente ajustado de mortalidade por DM de 3,4 e 7,2, por 10.000 habitantes, para o sexo masculino e feminino, respectivamente; para o mesmo período, entre japoneses residentes no Japão, esse coeficiente foi de 1,9 por 10.000 habitantes para ambos os sexos. Franco $^{3}$ destaca que maior risco de desenvolver o DM tem sido observado, por exemplo, entre migrantes japoneses residentes no Brasil e nos Estados Unidos da América, quando comparados àquele observado para o país de origem. A mesma experiência já havia sido registrada por outros investigadores ${ }^{5}$.

Ferreira e col. ${ }^{2}$, em harmonia com outros estudos, encontraram maior prevalência do DMNDI entre nipo-brasileiros residentes no interior do Estado de São Paulo, quando comparada com aquela observada no Japão. O encontro concomitante do DM com outros fatores de risco cardiovasculares (hipertensão e dislipidemia) possibilitou supor a existência de incremento na mortalidade desses indivíduos quando comparados àqueles que mantém a tolerância à glicose normal. Assim, como parte do estudo com esses migrantes japoneses, no presente trabalho são descritos e comparados os coeficientes de mortalidade observados em quatro anos de acompanhamento de indivíduos com diferentes graus de tolerância à glicose.

\section{MATERIAL E MÉTO DO}

Em 1993, a partir de um estudo de prevalência de DM entre migrantes japoneses e seus descendentes residentes na cidade de Bauru, no interior do Estado de São Paulo², 3 , foi formada uma coorte composta por 530 nipo-brasileiros de primeira (issei) e segunda geração (nisei), de ambos os sexos e com idade entre 40 e 79 anos. Nessa ocasião, utilizando-se o critério da $\mathrm{OMS}^{20}, 91$ indivíduos (17\%) foram classificados como diabéticos não dependentes de insulina (DMNDI), 90 (17\%) como portadores de tolerância à glicose diminuída (TGD) e 349 (66\%) como normais quanto à tolerância à glicose. Não houve diferenças estatisticamente significantes entre as prevalências de DM e TGD entre os sexos ${ }^{2,3}$.

Em 1996, a partir de uma lista utilizada para a atualização de endereços dos 530 indivíduos inicialmente incluídos no estudo, foi possível identificar os óbitos ocorridos durante esse período. Foram utilizadas para o registro da data e da causa das mortes as informações obtidas dos familiares e dos certificados de óbito. Obtiveram-se, para os três grupos de indivíduos, os coeficientes de mortalidade (CM) por todas as causas e por causas específicas ${ }^{18}$. Dentre as específicas foram selecionadas aquelas de natureza cardiovascular $\left(9^{\text {a }}\right.$ Revisão da Classificação Internacional de Doenças ${ }^{14}$ : capítulo de doenças circulatórias 390-459), uma vez que são as maiores responsáveis pelos óbitos em indivíduos diabéticos e também aquelas decorrentes de complicação renal ( $9^{\text {a }}$ Revisão da Classificação Internacional de Doenças $^{14}$ : capítulo de doenças do trato genito-urinário 580-599 - insuficiência renal crônica - 585), uma vez que acomete uma parcela significativa da população diabética ${ }^{16}$, ${ }^{22}$. Dado o fato de terem sido registrados apenas dois óbitos entre os indivíduos com TGD optou-se por não incluí-los nas etapas seguintes da análise.

As curvas de sobrevivência de indivíduos diabéticos e normais foram obtidas a partir da utilização da técnica de Kaplan-Meier ${ }^{9}$.

Foram consideradas variáveis de controle a idade, o sexo, a geração, o nível sérico de creatinina, a presença de hipertensão arterial, de dislipidemia e de obesidade (Tabela 1). A existência de associações entre essas variáveis e a ocorrência dos óbitos foi avaliada pela estatística quiquadrado.

O modelo de $\operatorname{Cox}^{9}$ foi utilizado para a comparação dos CM ajustados segundo as variáveis de controle (forma dicotômica). A variável idade foi incluída no modelo em sua forma contínua. Foi verificada a existência de possíveis interações entre as variáveis e estas foram mantidas no modelo final apenas quando o valor do respectivo coeficiente foi estatisticamente diferente de zero $(\mathrm{p}<0,05)$. Utilizou-se, em todas as etapas da análise, o programa Stata ${ }^{19}$. 
Tabela 1 - Forma categórica das variáveis consideradas na análise.

Table 1 - Categorical form of the variables considered in the analysis.

\begin{tabular}{|c|c|c|}
\hline Variável & Categoria & xposição \\
\hline Sexo & $\begin{array}{l}\text { Feminino } \\
\text { Masculino }\end{array}$ & $\begin{array}{r}\text { Basal } \\
\text { Exposto }\end{array}$ \\
\hline Idade & $\begin{array}{l}<60 \text { anos } \\
\geq 60 \text { anos }\end{array}$ & $\begin{array}{r}\text { Basal } \\
\text { Exposto }\end{array}$ \\
\hline Geração & $\begin{array}{l}\text { Primeira } \\
\text { Segunda }\end{array}$ & $\begin{array}{r}\text { Basal } \\
\text { Exposto }\end{array}$ \\
\hline O besidade & $\begin{array}{l}\text { Eutrófico }\left(I M C \leq 25 \mathrm{~kg} / \mathrm{m}^{2}\right) \\
\text { O beso }\left(I M C>25 \mathrm{~kg} / \mathrm{m}^{2}\right)\end{array}$ & $\begin{array}{r}\text { Basal } \\
\text { Exposto }\end{array}$ \\
\hline \multirow[t]{2}{*}{$\begin{array}{l}\text { Hipertensão } \\
\text { arterial }\end{array}$} & $\begin{array}{l}\text { Normotenso (pressão arterial sistólica } \leq 140 \mathrm{~mm} \mathrm{Hg} \text { e pressão arterial } \\
\text { diastólica } \leq 90 \mathrm{~mm} \mathrm{Hg} \text { e sem uso de medicação anti-hipertensiva) }\end{array}$ & Basal \\
\hline & $\begin{array}{l}\text { Hipertenso (pressão arterial sistólica }>140 \mathrm{~mm} \mathrm{Hg} \text { ou pressão } \\
\text { arterial diastólica }>90 \mathrm{~mm} \mathrm{Hg} \text { ou em uso de medicação anti-hipertensiva) }\end{array}$ & Exposto \\
\hline \multirow[t]{2}{*}{ Dislipidemia } & $\begin{array}{l}\text { Normal (colesterol total e triglicérides } \leq 200 \mathrm{mg} / \mathrm{dl} \text { e } \\
\mathrm{HDL} \text {-colesterol } \geq 35 \mathrm{mg} / \mathrm{dl} \text { e } \mathrm{LDL} \text {-colesterol } \leq 130 \mathrm{mg} / \mathrm{dl} \text { ) }\end{array}$ & Basal \\
\hline & $\begin{array}{l}\text { Dislipidêmico (colesterol total ou triglicérides }>200 \mathrm{mg} / \mathrm{dl} \text { ou } \\
\mathrm{HDL} \text {-colesterol }<35 \mathrm{mg} / \mathrm{dl} \text { ou LD L-colesterol > } 130 \mathrm{mg} / \mathrm{dl} \text { ) }\end{array}$ & Exposto \\
\hline Creatinina sérica & $\begin{array}{l}\text { Normal }(\leq 1,2 \mathrm{mg} / \mathrm{dl}) \\
\text { Alterado }(>1,2 \mathrm{mg} / \mathrm{dl})\end{array}$ & $\begin{array}{r}\text { Basal } \\
\text { Exposto }\end{array}$ \\
\hline
\end{tabular}

\section{RESU LTADOS}

Ao final de 1996, 507 (95,7\%) indivíduos estavam vivos. Dos 23 óbitos ocorridos durante o período de estudo, $13(56,5 \%)$ foram atribuídos a doenças circulatórias, um $(4,3 \%)$ a doença renal e nove $(39,2 \%)$ a causas diversas (acidentes, doença pulmonar obstrutiva crônica, pneumonia, leucemia e neoplasias de pâncreas, de intestino e de pulmão). Nove mortes foram observadas entre indivíduos diabéticos, sendo

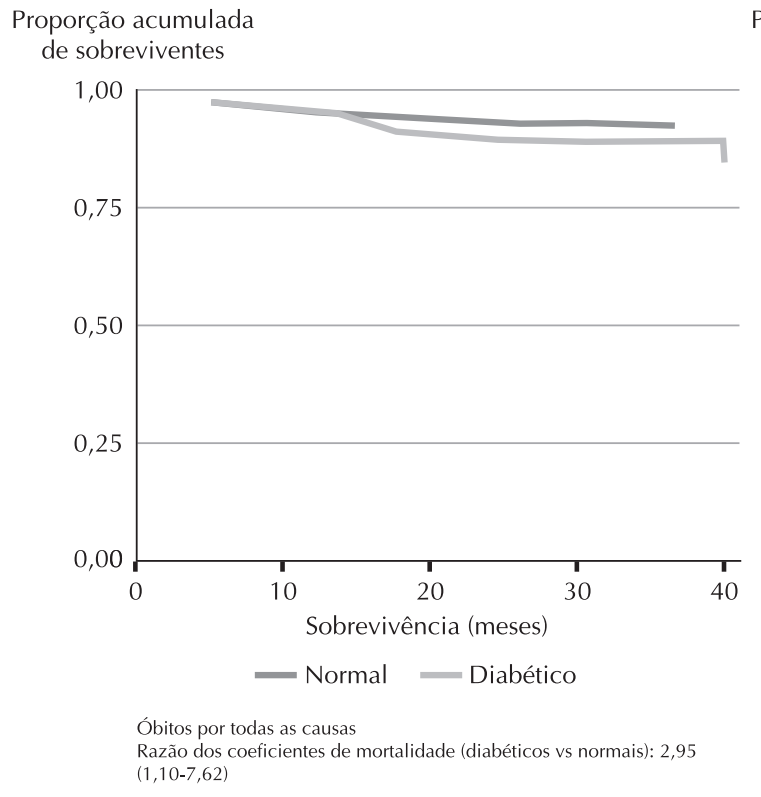

sete $(77,8 \%)$ por doenças circulatórias, uma $(11,1 \%)$ por doença renal e uma $(11,1 \%)$ por causa externa. Destaca-se que em apenas um certificado de óbito houve menção do DM entre as causas da morte.

Os CM brutos, tanto por todas as causas como por causas específicas, mostraram que a força de mortalidade foi maior entre os indivíduos diabéticos, tanto quando comparados aos indivíduos com TGD, como aos normais (Tabela 2). Esse fato é confirmado na Figura onde, em diferentes intervalos de tempo,

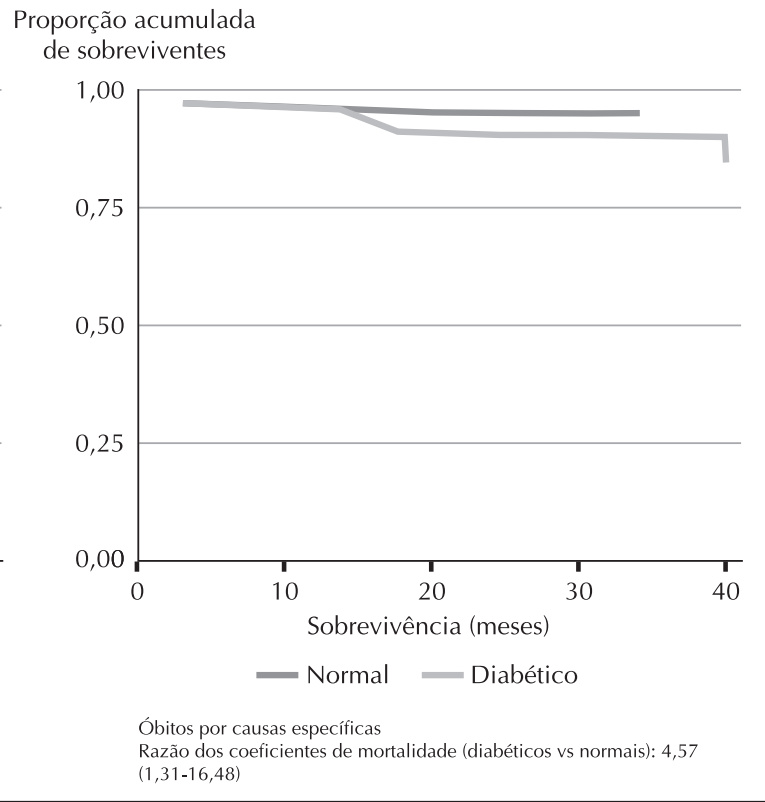

Figura - Proporção acumulada de sobrevivência (Kaplan-M eier) segundo grau de tolerância à glicose e causa do óbito. Figure - Kaplan-M eier survival curves by glucose tolerance status and causes of death. 
menor proporção de sobreviventes pôde ser observada entre os indivíduos diabéticos.

Observou-se, entre os que morreram (Tabela 3), proporção significativamente maior de nipo-brasileiros do sexo masculino ( $\mathrm{p}=0,003)$, com idade igual ou superior a 60 anos $(\mathrm{p}=0,027)$ e com nível sérico de creatinina aumentado $(\mathrm{p}=0,041)$. Esses indivíduos foram semelhantes àqueles que sobreviveram segundo a geração, presença de obesidade, de hipertensão arterial e de dislipidemia.

Na Tabela 4 estão apresentados os resultados finais obtidos a partir da utilização do modelo de Cox. Após

Tabela 2 - Tempo médio de sobrevivência, coeficiente de mortalidade bruto (CM) por todas as causas e por causas específicas segundo grau de tolerância à glicose.

Table 2 - Mean survival time, crude mortality rate for all causes and for circulatory and renal diseases according to glucose tolerance status.

\begin{tabular}{lccc}
\hline Grau de tolerância à glicose & $\begin{array}{c}\text { Tempo médio } \\
\text { de sobrevivência (meses) }\end{array}$ & $\begin{array}{c}\text { CM por todas as causas } \\
\text { (por 1.000 pessoas-ano) }\end{array}$ & $\begin{array}{c}\text { CM por causas específicas } \\
\text { (por 1.000 pessoas-ano) }\end{array}$ \\
\hline Diabetes & 20,0 & 31,5 & 24,7 \\
Tolerância à glicose diminuída & 21,7 & 6,9 & 3,4 \\
N ormal & 21,9 & 10,7 & 5,4 \\
\hline
\end{tabular}

Tabela 3 - N úmero e percentage de nipo-brasileiros residentes em Bauru, SP, segundo características demográficas e biológicas.

Table 3 - Number and percentage of Japanese-Brazilians in Bauru, SP by demographic and biological characteristics.

\begin{tabular}{|c|c|c|c|c|c|c|c|c|c|c|c|}
\hline \multirow{4}{*}{ Variável } & \multicolumn{8}{|c|}{ Condição em 1996} & & & \\
\hline & \multicolumn{4}{|c|}{ Vivo } & \multicolumn{4}{|c|}{ Morto } & & & \\
\hline & \multicolumn{2}{|c|}{ Diabético } & \multicolumn{2}{|c|}{ Normal } & \multicolumn{2}{|c|}{ Diabético } & \multicolumn{2}{|c|}{ Normal } & \multicolumn{2}{|c|}{ Total } & \multirow[b]{2}{*}{ Q ui-quadrado (p) } \\
\hline & $\mathrm{N}$ & $\%$ & $\mathrm{~N}$ & $\%$ & $\mathrm{~N}$ & $\%$ & $\mathrm{~N}$ & $\%$ & $\mathrm{~N}$ & $\%$ & \\
\hline \multicolumn{12}{|l|}{ Sexo } \\
\hline M asculino & 48 & 58,5 & 154 & 45,7 & 9 & 100 & 8 & 66,7 & 219 & 49,8 & $8,6(0,003)$ \\
\hline Feminino & 34 & 41,5 & 183 & 54,3 & - & - & 4 & 33,3 & 221 & 50,2 & \\
\hline Total & 82 & 100 & 337 & 100 & 9 & 100 & 12 & 100 & 440 & 100 & \\
\hline \multicolumn{12}{|l|}{ Idade } \\
\hline$<60$ anos & 30 & 36,6 & 193 & 57,3 & 2 & 22,2 & 4 & 33,3 & 229 & 52,0 & $4,9(0,027)$ \\
\hline$\geq 60$ anos & 52 & 63,4 & 144 & 42,7 & 7 & 77,8 & 8 & 66,7 & 202 & 48,0 & \\
\hline Total & 82 & 100 & 337 & 100 & 9 & 100 & 12 & 100 & 440 & 100 & \\
\hline \multicolumn{12}{|l|}{ G eração } \\
\hline Primeira & 41 & 50,0 & 145 & 43,0 & 5 & 55,6 & 6 & 50,0 & 197 & 44,9 & $0,5(0,472)$ \\
\hline Segunda & 41 & 50,0 & 192 & 57,0 & 4 & 44,4 & 6 & 50,0 & 243 & 55,1 & \\
\hline Total & 82 & 100 & 337 & 100 & 9 & 100 & 12 & 100 & 440 & 100 & \\
\hline \multicolumn{12}{|l|}{ Estado nutricional } \\
\hline Eutrófico & 37 & 45,1 & 205 & 60,8 & 6 & 66,7 & 9 & 75,0 & 257 & 58,4 & \\
\hline O beso & 38 & 46,3 & 110 & 32,6 & 2 & 22,2 & 2 & 16,7 & 152 & 34,5 & $2,2(0,137)$ \\
\hline Sem informação & 7 & 8,6 & 22 & 6,6 & 1 & 11,1 & 1 & 8,3 & 31 & 7,1 & \\
\hline Total & 82 & 100 & 337 & 100 & 9 & 100 & 12 & 100 & 440 & 100 & \\
\hline \multicolumn{12}{|l|}{ Pressão arterial } \\
\hline Normotenso & 44 & 53,7 & 264 & 78,3 & 4 & 44,4 & 9 & 75,0 & 321 & 73,0 & \\
\hline Hipertenso & 38 & 46,3 & 71 & 21,1 & 5 & 55,5 & 3 & 25,0 & 117 & 26,6 & $1,5(0,227)$ \\
\hline Sem informação & - & - & 2 & 0,6 & - & - & - & - & 2 & 0,4 & \\
\hline Total & 82 & 100 & 337 & 100 & 9 & 100 & 12 & 100 & 440 & 100 & \\
\hline \multicolumn{12}{|l|}{ Perfil lipídico } \\
\hline Normal & 7 & 8,5 & 76 & 22,6 & 2 & 22,2 & 3 & 25,0 & 88 & 20,0 & $0,2(0,655)$ \\
\hline Dislipidêmico & 75 & 91,5 & 261 & 77,4 & 7 & 77,8 & 9 & 75,0 & 352 & 80,0 & \\
\hline Total & 82 & 100 & 337 & 100 & 9 & 100 & 12 & 100 & 440 & 100 & \\
\hline \multicolumn{12}{|l|}{ Creatinina } \\
\hline Normal & 66 & 80,5 & 299 & 88,7 & 6 & 66,7 & 9 & 75,0 & 380 & 86,4 & $4,2(0,041)$ \\
\hline Alterada & 16 & 19,5 & 38 & 11,3 & 3 & 33,3 & 3 & 25,0 & 60 & 13,6 & \\
\hline Total & 82 & 100 & 337 & 100 & 9 & 100 & 12 & 100 & 440 & 100 & \\
\hline
\end{tabular}


Tabela 4 - Resultados da análise com o modelo de Cox (modelo final).

Table 4 - Final Cox models for deaths by all causes and for circulatory and renal diseases.

\begin{tabular}{|c|c|c|c|}
\hline Ó bitos & Variável & RDI* & IC $95 \%$ \\
\hline \multicolumn{4}{|l|}{ Ó bitos por } \\
\hline \multirow[t]{6}{*}{ todas as causas } & Diabetes & 1,61 & $0,62-4,17$ \\
\hline & Sexo & 5,56 & $1,72-16,67$ \\
\hline & Idade & 1,06 & $1,01-1,12$ \\
\hline & Hipertensão arterial*dislipidemia & 0,05 & $0,01-0,27$ \\
\hline & Hipertensão arterial & 13,89 & $3,64-53,00$ \\
\hline & Dislipidemia & 1,10 & $0,33-3,68$ \\
\hline \multicolumn{4}{|l|}{ Ó bitos por } \\
\hline \multirow[t]{4}{*}{ causas específicas } & Diabetes & 3,86 & $1,11-13,38$ \\
\hline & Sexo & 9,09 & $1,16-100,00$ \\
\hline & Idade & 1,04 & $0,97-1,12$ \\
\hline & O besidade & 0,34 & $0,07-1,65$ \\
\hline
\end{tabular}

* RDI: razão de densidades de incidência ou razão dos coeficientes de mortalidade

IC - Intervalo de confiança

o ajuste simultâneo do efeito às covariáveis, observase que, entre os indivíduos diabéticos, a força de mortalidade (RDI) por causas específicas foi aproximadamente quatro vezes aquela observada entre os indivíduos normais ( $\mathrm{RDI}=3,86$ IC 95\%: 1,1113,38). Para os óbitos ocorridos por todas as causas, as diferenças entre os CM ajustados de indivíduos com DMNDI e o dos indivíduos normais não foram estatisticamente significantes.

\section{DISCUSSÃO}

Geiss e col. ${ }^{6}$ comentaram que, em estudos de mortalidade, o delineamento prospectivo oferece algumas vantagens sobre aqueles que utilizam exclusivamente, como fonte de dados, os certificados de óbito. Os estudos prospectivos, em geral, permitem: 1) descrever a mortalidade de todos os indivíduos diabéticos e não apenas daqueles que tiveram o DM mencionado no certificado de óbito; 2) comparar o risco de morrer observado entre diabéticos e não diabéticos; 3) examinar os fatores de risco, para a mortalidade, não disponíveis nos documentos oficiais. Entre suas limitações, os autores destacaram: 1) a maioria dos estudos de coorte não fazem distinção entre mortalidade por DM dependente de insulina e por DMNDI; 2) em geral, esses estudos são feitos com um pequeno número de indivíduos diabéticos; 3) alguns identificam os portadores de DM apenas no início do estudo, levando a uma subestimação do impacto do DM sobre a mortalidade.

O delineamento utilizado nesse estudo permitiu descrever e comparar, controlando-se o efeito de outros fatores de risco, os CM de indivíduos com diferentes graus de tolerância à glicose. Se alguma interferência ocorreu nas medidas de efeito obtidas, essas foram no sentido de subestimar a importância do DM sobre a mortalidade, dado que os portadores de DMNDI foram identificados apenas no início do estudo.

Aimportante parcela de contribuição para a mortalidade geral, em diferentes populações, das doenças do aparelho circulatório e de sua associação com o DM está bem documentada na literatura $4,6,21,22$. No presente estudo, $56,5 \%$ do total de óbitos foram decorrentes dessas doenças. Essa cifra elevou-se para $77,8 \%$ quando se consideraram apenas os óbitos ocorridos entre indivíduos diabéticos. Verificou-se também a ausência de menção, nos documentos oficiais, do DM como causa do óbito, refletindo, em nosso meio, a importante subnotificação dessa doença como causa de morte.

A maior força de mortalidade, bruta e ajustada, observada entre os indivíduos nipo-brasileiros diabéticos (Tabelas 2 e 4), é consistente com os relatos de outros em diferentes populações ${ }^{1,12}$. Geiss e col. ${ }^{6}$ destacam que indivíduos diabéticos têm aproximadamente o dobro da força de mortalidade observada entre aqueles sem a doença. No presente estudo, ao considerar os óbitos ocorridos por todas as causas (Tabela 4), a razão dos coeficientes de mortalidade foi de 1,61 que, apesar de não ter sido estatisticamente significante (IC 95\%: 0,62 - 4,17), aproximouse dos valores encontrados por outros pesquisadores.

Geiss e col. ${ }^{6}$ comentam que os resultados publicados evidenciam a força de mortalidade por doenças circulatórias entre indivíduos diabéticos, que é cerca de duas a quatro vezes aquela observada entre indivíduos sem a doença. Em harmonia com esses achados, ao se considerar os óbitos decorrentes de causas específicas, foi possível detectar no presente estudo 
diferenças estatisticamente significantes entre as experiências de mortalidade de indivíduos com DMNDI e de normais (RDI: 3,86; IC 95\%: 1,11 13,38), confirmando também, entre nipo-brasileiros, a importância do DM como causa de morte, independente de outros fatores de risco.

Os presentes achados, mesmo que para um

\section{REFERÊNCIAS}

1. CARTER, J.S.; WIGGINS, C.L.; BECKER, T.M.; KEY, C.R.; SAMET, J.M. Diabetes mortality among New Mexico American Indian, Hispanic, and Non-Hispanic populations, 1958-1987. Diabetes Care, 16: 306-9, 1993.

2. FERREIRA, S.G.; IUNES, M.; FRANCO, L.J.; IOCHIDA, L.C.; HIRAI, A.; VIVOLO, M.A.; JAPANESE-BRAZILIAN DIABETES STUDY GROUP. Disturbances of glucose and lipid metabolism in first and second generation Japanese-Brazilians. Diabetes Res. Clin. Pract., 34: 59-63, 1996.

3. FRANCO, L.J. Diabetes in Japanese-Brazilians - influence of the acculturation process. Diabetes Res. Clin. Pract., 34: 517, 1996.

4. FRANCO, L.J.; IOCHIDA, L.C.; NAMERI, C.; PAGLIARO, H.; FERREIRA, S.R.G. De que morrem os diabéticos no Estado de São Paulo? Análise de causas múltiplas de óbito em 1992. Rev. Assoc. Lat.-Am. Diabetes, 3: 87, 1995 [abstract 35].

5. FUJIMOTO, W.Y. The growing prevalence of non-insulindependent diabetes in migrant Asian populations and its implications for Asia. Diabetes Res. Clin. Pract., 15: 167-84, 1992.

6. GEISS, L.S. \& HERMAN, W.H. Mortality in non-insulindependent diabetes. In: Diabetes in America. 2nd ed., Bethesda, National Institutes of Health, chapter 11, p. 23357. 1995. (NIH Publication 95-1468).

7. GOTLIEB, S.L.D. Mortalidade em migrantes japoneses residentes no Município de São Paulo. Rev. Saúde Pública, 24: 453-67, 1990.

8. HANIS, C.L.; CHU, H.L.; LAWSON, K.; HEWETTEMMETT, D.; BARTON, S.A.; SCHULL, W.J.; GARCIA, C.A. Mortality of Mexican Americans with NIDDM. Diabetes Care, 16: 82-9, 1993.

9. HARRIS, E.K. \& ALBERT, A. Survivorship analysis for clinical studies. New York, Marcel Dekker, 1991.

10. KAPLAN, N.M. The deadly quartet: upper-body obesity, glucose intolerance, hypertriglyceridemia and hypertension. Arch. Intern. Med., 149: 1514-20, 1989.

11. LAURENTI, R.; FONSECA, L.A.M.; COSTA J.R., M.L. Mortalidade por diabetes mellitus no Município de São período de seguimento relativamente curto (quatro anos), confirmam a necessidade de adoção de medidas que visem tanto à prevenção como ao controle do DM que, nas últimas décadas, vem se destacando como uma das doenças crônicas que mais tem alterado o perfil de morbimortalidade das populações em todo o mundo.
Paulo (Brasil): evolução em um período de 79 anos (19001978) e análise de alguns aspectos sobre associação de causas. Rev. Saúde Pública, 16: 77-91, 1982.

12. LEE, E.T.; RUSSEL, D.; KENNY, S.; YU., M.L. A followup study of diabetic Oklahoma Indians: mortality and causes of death. Diabetes Care, 16: 300-5, 1993.

13. LESSA, I. Tendência da mortalidade proporcional pelo diabetes mellitus nas capitais brasileiras, 1950-1985. Bol. Oficina Sanit. Panan., 113: 212-7, 1992.

14. MANUAL DA CLASSIFICAÇÃO ESTATÍSTICA INTERNACIONAL DE DOENÇAS, LESÕES E CAUSAS DE ÓBITO: $9^{a}$ revisão 1975. São Paulo, Centro da OMS para Classificação de Doenças em Português/Organização Panamericana de Saúde, 1980.

15. MELO, M.S.; LÓLIO, C.A.; LUCENA, M.A.F.; KIRZNER, C.F.; MARTINS, S.M.; BARROS, M.N.D.S. Causas múltiplas de morte em diabéticos no Município de Recife, 1987. Rev. Saúde Pública, 25: 435-42, 1991.

16. NELSON, R.G.; KNOWLER, W.C.; PETTITT, D.J.; BENNETT, P.H. Kidney diseases in diabetes In: Diabetes in America, 2nd ed., Bethesda, National Institutes of Health, chapter 16, p. 349-400. 1995. (NIH Publication, 95-1468).

17. NEWMAN, J.M.; DESTEFANO, F.; VALWAY, S.E.; GERMAN, R.R.; MUNETA, B. Diabetes-associated mortality in Native Americans. Diabetes Care, 16: 297-9, 1993.

18. ROTHMAN, K.J. Modern epidemiology. Boston, Little Brown and Company, 1986.

19. STATACORP: Stata statistical software: release 4.0. College Station, TX Stata Corporation, 1995.

20. WHO Study Group . Diabetes mellitus: report. Geneva, World Health Organization, 1985. (WHO Technical Report Series, 727), 1985.

21. WHO Study Group. Prevention of diabetes mellitus. Geneva, World Health Organization, 1994. (WHO Technical Report Series, 844).

22. WINGARD, D.L. \& BARRETT-CONNOR, E. Heart disease and diabetes. In: Diabetes in America. 2nd ed. chapter 19, 429-48. 1995. (NIH Publication, 95-1468). 